\title{
The Human Lineage
}

Matt Cartmill and Fred H. Smith

Hoboken, NJ: Wiley-Blackwell, 2009, 624 pp. (hardback), \$109.95.

ISBN-13: 9780471214915.

\section{Reviewed by ISABELLE WINDER}

Department of Archaeology, The King's Manor, University of York, York YO1 7EP, UNITED KINGDOM; icw503@york.ac.uk

$\mathrm{T}_{\mathrm{D}}$ he year 2009 marked both the bicentennial of Charles Darwin's birth and the sesquicentennial of the publication of his most famous book, On the Origin of Species. The response to this historical milestone was a proliferation of evolutionary research and writing, producing a multitude of television series, news articles, popular science books, textbooks, and special issue academic journals. Cartmill and Smith's The Human Lineage, published as part of the "Foundations of Human Biology" series, can be seen as one of the more lasting contributions to this evolutionary proliferation.

This textbook, aimed at advanced undergraduates and postgraduates in paleoanthropology courses, tackles a rather difficult task - that of presenting the substantial body of paleontological, genetic, geological and archaeological evidence regarding human evolution, and the associated scientific history, in a logical and readable way without sacrificing either clarity or detail. Although paleoanthropology as a field moves rapidly - with major events like the publication of Ardipithecus ramidus and "X-woman," and the discovery of Australopithecus sediba occurring just in the year since The Human Lineage was publishedthe sheer quality of the writing and explanatory synthesis in this book will undoubtedly make it a valuable resource for students for many years.

The book is split into two main parts, an introductory section comprising three chapters, and a main body of five chapters, chronologically ordered to progress through hominin evolutionary history. For some readers, the introductory material covered in Section One will seem too elementary. These chapters deal with three key topics - the fossil and geological record (Chapter One), the nature of evolution (Chapter Two) and the primate order (Chapter Three). In fact, the pace of these chapters does pick up, and while they provide a relatively gentle ramble through the history of geology, evolutionary biology, and life on Earth, most student readers at least will encounter new material at some point.

Chapter One begins with the history of geological ideas and scientific understanding of the fossil record. The latter part of that chapter covers the history of life from its unicellular origins to the anthropoid radiations, providing much more detail than is typical of paleoanthropologically focused texts. Chapter Two begins with a history of evolutionary thought, before delving into the debates over species concepts, speciation, phylogenetics, and evolutionary patterns, illustrated with biological examples but nonethe- less covering material from such fields as evolutionary genetics, systematics, and biological philosophy at substantial length. To cap this primer section, Cartmill and Smith then provide a chapter entitled People as Primates, which not only summarizes the evolution of our order but also discusses the anatomical basis of classification, the relationships of primates to other orders, and the characteristics of extant primate groups, even those only distantly relevant to the direct line of hominin history.

The importance of this primer will be most obvious to students new to paleoanthropology and evolutionary primatology. The level of detail and scope of the three background chapters, which together cover some 128 pages, is such that they provide a very holistic introduction to the subject, if only because many paleoanthropology and primatology courses do not discuss the early history of life as this book does. Those interested in paleoanthropology's sister-disciplines-taxonomy, paleontology, evolutionary biology, or anatomy, for example-also will find this section useful for reference. Later sections of The Human Lineage make use of the evidence and contextual information presented in the early chapters, and the book's first section provides a useful way into the relevant literature for those unfamiliar with the subject(s) involved.

The second section, Chapters Four through Eight, comprises the main body of the book. These chapters each take as their title one of the major episodes in human evolutionary history, beginning with the "bipedal apes" (the australopithecines and their Plio-Pleistocene relatives), and continuing through the "migrating apes" (Homo erectus), "big-brained apes," "talking apes," and, finally, "symbolic apes" (Homo sapiens). Within chapters, though, treatment of subject matter is not strictly chronological. In the bipedal ape chapter, for example, the australopithecines-as the better known and more widely studied "early humans" are presented first, with the evidence for taxa like Sahelanthropus, Orrorrin, and Ardipithecus given only afterward. Instead, the apes of the titles are used as starting points for discussion of particular themes and debates in modern paleoanthropology, with brief scientific histories and "blind alley" sections used to describe past controversies or resolved debates in paleoanthropological research.

Rather than emphasize a particular hominin taxonomy to the exclusion of others, Cartmill and Smith have chosen the simpler route of avoiding taxonomic debate where it is not under direct discussion. Instead of using widely debated species or taxon names, then, they opt for more informal 
descriptions of fossil groups. Thus, while the debate over Homo ergaster/erectus is certainly covered in the "migrating ape" chapter, it is restricted to certain sub-sections of the chapter, and in the main body the hominins concerned are referred to as "erectines" (for all relevant fossils) or "ergasts" (for the earliest specimens only). This informal terminology not only minimizes the intrusion of contrasting taxonomies in discussion of issues like dispersal, cognitive evolution, cultural development, and encephalization, it also helps to make the text more readable, by cutting out phrases like "those specimens referred to" and "putative specimens of" that can otherwise become common in paleoanthropological textbooks.

The authors' taxonomic opinions are not invisible, however. Homo habilis and $H$. rudolfensis, the taxa referred to as "habilines," are placed quite firmly in the "bipedal ape" chapter with australopithecine-grade taxa; all the paranthropine taxa are referred to as members of Australopithecus, and there is considerable scepticism over the taxonomy of several Pliocene hominins within the text. However, these taxonomic opinions are not the central focus of the discussion, which instead centers on the nature of the evidence supporting (and standing against) particular interpretations, so that the reader has to make up their own mind whether they agree with the authors' classifications.

Interestingly, the talking ape that titles Chapter Seven is not our own species, but the Neandertal, although the conclusion of the section on language is nonetheless that "no aspect of skeletal anatomy can tell us whether Neandertals - or any other ancient humans - had humanlike capacities for making and using language" (page 395). The discussion instead turns to the archaeological evidence for symbolic behaviors - art and religion. While no firm conclusions can be drawn there either, the authors' discussion of the Neandertals is characteristic of their tone throughout the book - they present both the history of the lineage and the history of its study, and maintain a relatively neutral position regarding most ongoing debates. In all cases, they present the evidence for and against all the major positions in each disagreement as well as, or in place of, their own opinion.

The subject matter of this second part of the book varies widely, covering ground in anatomy, ecology, archaeology, genetics, the study of culture, and environmental science. As a result, each chapter is fairly long, with the shortest, Chapter Six on Middle Pleistocene hominins (Heidelbergs), coming in at 46 pages, too long to be easily read in a single sitting. The splitting of material into short chunks, then, is an absolute necessity. The text does not use numerical headings, and so minimizes the internal cross-referencing that spoils so many textbooks. The downside, of course, is that it becomes a little harder to track themes through multiple chapters, but the book has a 44-page index that proved its worth on several occasions.

One of The Human Lineage's major selling points is likely to be the illustration. In what must have been a substantial undertaking, all the book's pictures of hominin fossils have been re-drawn in great detail as black and white stipple drawings, giving the whole volume a coherent style and vastly improving the appearance of many specimens often presented in relatively old photographs or low-resolution line drawings. Whether these pictures have been created from the original fossils or photographs, the quality of the finished product is excellent, and their inclusion adds significantly to the pleasure of reading as well as contributing materially to understanding the text. In a few cases, however, these images seem to be over-reduced to fit on the page, making the level of contrast between features lower. Despite this, the key features remain much more visible in these drawings than they would have been on most photographs, and they are much more attractive to the reader than the disparate and sometimes low-quality illustrations they replace. This must have taken a lot of time and the book clearly benefits from that investment.

Finding downsides to The Human Lineage, in fact, is difficult. The price, at $\$ 109.95$, may at first glance appear off-putting, but the book's quality and comprehensive coverage offset this. Where many paleoanthropology courses require students to purchase several books, each emphasising a different line of evidence, taxonomic group, theoretical perspective, or time period, The Human Lineage could be used as a sole text, supplemented with journal articles and library sources. At this price, however, it is unlikely to penetrate a wider market, which is a shame. The quality of writing, illustration, and presentation of material, together with the comprehensive background section, are such that the book would be accessible to readers inspired by the Darwiniana of this bicentennial year. The market for readable professional texts has been well-prepared, but non-specialist readers will probably balk at the price, notwithstanding the book's size and quality. Perhaps one way around this would be to publish a paperback edition. 